\title{
Resección de adenoma velloso gigante causante del síndrome de Mckittrick-Wheelock
}

\author{
Marcos Leites, ${ }^{1}$ Adrián Canavesi, ${ }^{1}$ Diana Valencia, ${ }^{1}$ Rodrigo Dorelo, ${ }^{1}$ Iván Trostchansky, ${ }^{2}$ \\ Marta Otero, ${ }^{3}$ César Canessa ${ }^{4}$ \\ ${ }^{1}$ Clinica de Gastroenterología "Prof. Dra. Carolina Olano". \\ ${ }^{2}$ Clínica quirúrgica F "Prof. Dr. Roberto Valiñas". \\ ${ }^{3}$ Cátedra de Anatomía Patológica "Prof. Dr. Dardo Centurión". \\ ${ }^{4}$ Clínica quirúrgica B "Prof. Dr. César Canessa". \\ Hospital de Clinicas "Dr. Manuel Quintela”, Montevideo, Uruguay.
}

Acta Gastroenterol Latinoam 2021;51(1):93-99

Recibido: 07/12/2020 / Aceptado: 16/02/2021 / Publicado online: 22/03/2021 / https://doi.org/10.52787/rwop3269

\section{Resumen}

Los adenomas vellosos colorrectales de gran tamaño son excepcionales. Pueden causar una rectorragia, una diarrea crónica $y$, de forma menos frecuente, una obstrucción y un prolapso rectal. Además, pueden secretar un material mucinoso rico en electrolitos, ocasionando alteraciones hidroelectroliticas, la deshidratación e injuria renal aguda. El manejo de estos adenomas por su tamaño ha sido quirúrgico en la mayor parte de los casos. A continuación, se presenta y discute el caso de un hombre de 73 años que consultó por sincope, diarrea crónica y alteración hidroelectrolítica. En la colonoscopia se observó una lesión de crecimiento lateral que comprometía circunferencialmente todo el recto. La histología correspondió a un adenoma velloso con displasia de bajo grado. Se corrigió la injuria renal y el desequilibrio hidroelectrolitico con un posterior tratamiento quirúrgico mediante la técnica del pull through con anastomosis coloanal.
Correspondencia: Marcos Leites

Residente, Hospital de Clinicas "Dr. Manuel Quintela". Montevideo, Uruguay Correo electrónico: mleitesmadera@gmail.com
Palabras claves. Adenoma velloso gigante, sindrome de Mckittrick-Wheelock, alteración hidroelectrolitica, insuficiencia renal aguda, cirugía transanal.

\section{Resection of a Giant Villous Adenoma Causing Mckittrick-Wheelock Syndrome}

\section{Summary}

Large colorectal villous adenomas are unusual. They can cause a rectal bleeding, a chronic diarrhea and less frequently a rectal obstruction and a prolapse. In addition, they can secrete a mucinous material rich in electrolytes, causing hydroelectrolyte disturbances, dehydration and acute kidney injury. In most cases, the management of these adenomas due to their size has been surgical, but endoscopic treatment has been reported with promising results, reducing the morbidity. We report and discuss the case of a 73-year-old man, who presented with syncope, with chronic diarrhea and a hydroelectrolytic alteration. Colonoscopy revealed a granular lateral spreading tumor of the rectum, and pathology confirmed a villous adenoma with a low-grade dysplasia. An initial symptomatic management of diarrhea and electrolyte disorders was performed with a subsequent surgical treatment using pull through technique with coloanal anastomosis.

Keywords. Giant Villous adenoma, Mckittrick-Wheelock Syndrome, hidroelectrolyte disorders, acute kidney injury, pull through surgery.

\section{Abreviaturas}

LST: Tumor de crecimiento lateral (lateral spreading tumor). 
SMcW: Sindrome de Mckittrick Wheelock.

PGE2: Prostaglandina E2.

AMPc: Adenosin Monofosfato cíclico.

VCC: Videocolonoscopía.

REM: Resección endoscópica mucosa.

DES: Disección endoscópica submucosa.

USE: Ultrasonografía endoscópica.

TEM: Microcirugía transanal endoscópica.

TAMIS: Cirugía transanal minimamente invasiva.

TaTME: Escisión transanal mesorrectal total.

\section{Introducción}

Los pólipos planos se definen endoscópicamente como lesiones no exofíticas, planas o deprimidas, con una altura menor a $2,5 \mathrm{~mm}$. Cuando se extienden más de $10 \mathrm{~mm}$ y presentan una altura menor a la mitad del diámetro, se denominan tumores de crecimiento lateral (LST: lateral spreading tumor). ${ }^{1,2}$

Los LST se caracterizan por presentar un alto índice de apoptosis celular y una baja proliferación en capas profundas, lo que le confiere la propagación lateral y poca frecuencia de invasión en profundidad, incluso después de la transformación a adenocarcinoma. ${ }^{3}$

Los LST granulares son de los más frecuentes en la mayoría de las publicaciones. Sin embargo, algunos artículos mencionan a los no granulares como los más frecuentes, argumentando que estos suelen ser subdiagnosticados. ${ }^{3}$

En el 2013, Yusuke Horiuchi et al. realizaron un estudio en el que concluyeron que los LST no granulares tienen un mayor riesgo de cáncer e invasión submucosa. La probabilidad de esta última crece conforme aumenta el tamaño del tumor. ${ }^{4}$

El 5\% de los adenomas corresponde al tipo velloso. Cuando se localizan en el recto suelen ser de gran tamaño. ${ }^{5,6}$ Los menores de $10 \mathrm{~mm}$ tienen 10 veces más riesgo de transformarse en carcinomas invasores que los adenomas tubulares del mismo tamaño y cuando el diámetro es mayor a $20 \mathrm{~mm}$ el riesgo de malignización es del 50\%.7

La mayor parte de los LST vellosos de recto no presentan síntomas. Ocasionalmente pueden debutar con tenesmo, rectorragia y, en casos muy raros, con diarrea crónica, deshidratación y alteración hidroelectrolítica denominándose síndrome Mckittrick Wheelock $(\mathrm{SMcW})$. 5, 6, 8, 9

El tratamiento inicial consiste en la reposición hidroelectrolítica, la estabilización hemodinámica, el control de la diarrea y la resección endoscópica o quirúrgica del adenoma rectal como tratamiento definitivo. ${ }^{5}$

\section{Caso clínico}

Un hombre de 73 años es alcohólico y tabaquista en abstinencia. Consultó al servicio de emergencias por síncope. Refirió una diarrea de seis meses de evolución, de consistencia líquida, con 4 a 6 deposiciones por día, de escaso volumen, acompañada de pujos y tenesmo rectal, sin otra sintomatología digestiva ni sistémica.

En el examen físico se constató un paciente lúcido, apirético, con un buen estado nutricional y un examen pleuropulmonar normal. Se observaron elementos clínicos de deshidratación dados por la piel y las mucosas secas, el pliegue hipoelástico, el relleno capilar lento y la frecuencia cardíaca de 100 ciclos por minuto.

El examen abdominal fue normal. En el tacto rectal estaba el esfínter normotónico, la mucosa granular con un compromiso circunferencial y había una escasa cantidad de moco y sangre en el guante.

En el análisis bioquímico se constató una insuficiencia renal con un aumento de azoemia, creatininemia y filtrado glomerular de $25 \mathrm{ml} / \mathrm{min} / 1,73 \mathrm{~m}^{2}$; hiponatremia, hipokalemia severa e hipocloremia (Tabla 1).

Se inició la reposición hidroelectrolítica con suero fisiológico y potasio intravenoso con una buena respuesta.

Se le realizó una videocolonoscopía (VCC), donde se observó LST de tipo granular no homogéneo, que ocupaba desde el margen anal hasta los $18 \mathrm{~cm}$ de forma circunferencial y continua.

Se realizó una cromoendoscopía digital (NBI Olympus $\left.{ }^{\circledR}\right)$ con un zoom óptico hasta $1,5 x$ donde se observó un patrón cerebroide, tipo IV de la clasificación de Kudo, sugestivo de adenoma velloso (Figura 1 y Video). Como un elemento sugestivo de invasión submucosa se evidenció un nódulo predominante. Se hizo una biopsia e informó histológicamente como un adenoma velloso con una displasia de bajo grado (Figura 2).

\section{Tabla 1}

\begin{tabular}{lcc}
\hline & Resultado & Valores de referencia \\
\hline Hemoglobina $(\mathrm{g} / \mathrm{dl})$ & 16,9 & $13,4-16,6$ \\
Plaquetas $\left(\mathrm{ml} / \mathrm{mm}^{3}\right)$ & 414 & $130-400$ \\
Azoemia $(\mathrm{mg} / \mathrm{dl})$ & 168 & $12,8-42,80$ \\
Creatinina $(\mathrm{mg} / \mathrm{dl})$ & 2,5 & $0,70-1,20$ \\
Sodio $(\mathrm{mEq} / \mathrm{l})$ & 124 & $136-145$ \\
Potasio $(\mathrm{mEq} / \mathrm{l})$ & 2,2 & $3,5-5,1$ \\
Cloro $(\mathrm{mEq} / \mathrm{l})$ & 73 & $98-107$
\end{tabular}


Figura 1. Endoscopía
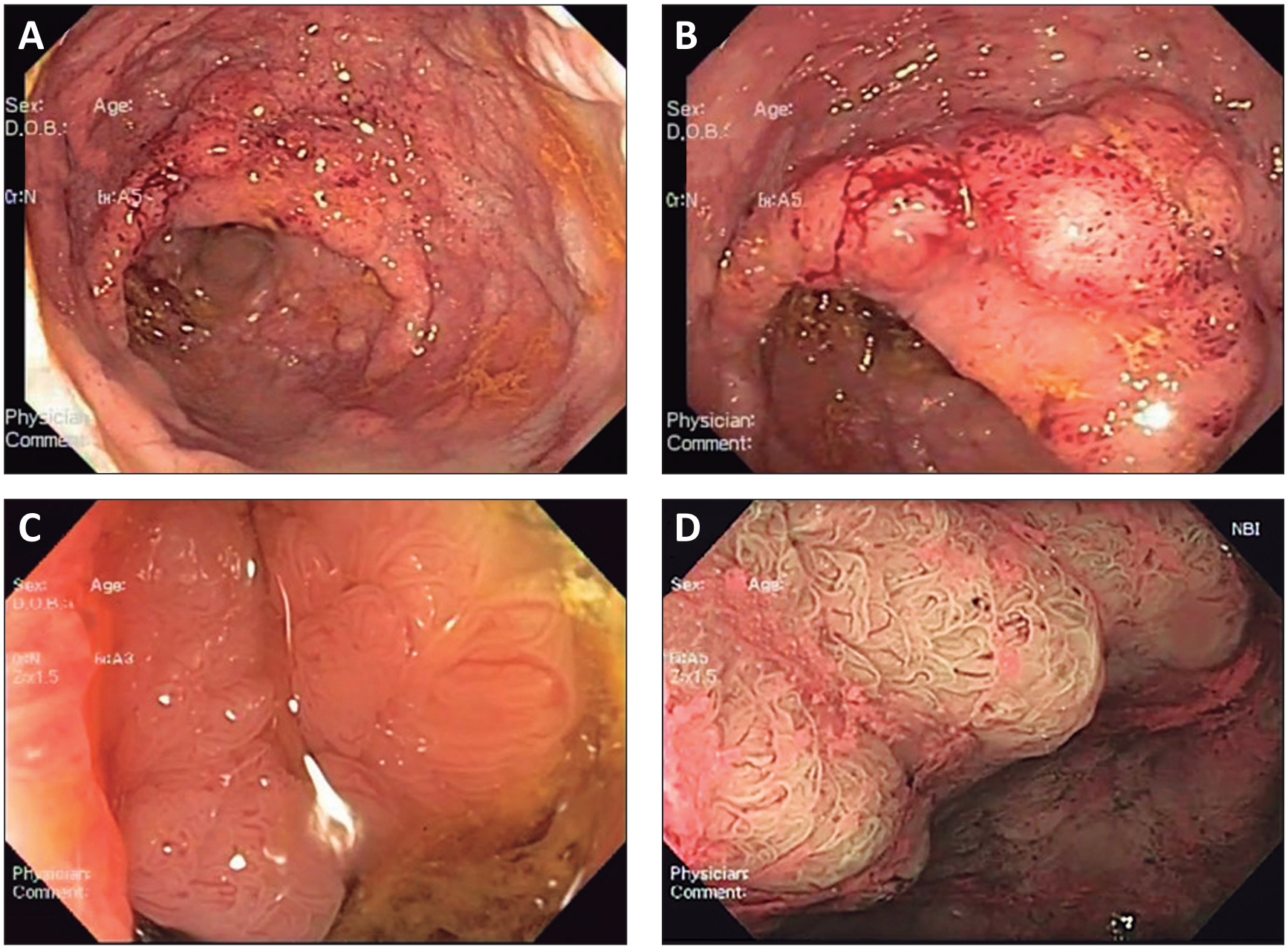

A: LST nodular mixto del recto con un compromiso circunferencial desde el margen anal hasta los $18 \mathrm{~cm}$ proximales.

B: Nódulo dominante visto con luz blanca y alta definición.

C: Visión con zoom óptico 1,5x donde se observa el patrón glandular ramificado.

D: Visión con zoom óptico 1,5x + cromoendoscopía digital (NBI Olympus $®)$ donde se observa un patrón glandular cerebroide (Kudo IV) que sugiere un adenoma velloso.

Video. Puede visualizarse en el siguiente enlace: https://www.youtube.com/ watch? $v=Y G J U P-7 U$ sdoerfeature $=e m b \_t i t l e b a b \_c h a n n e l=$ ActaSage

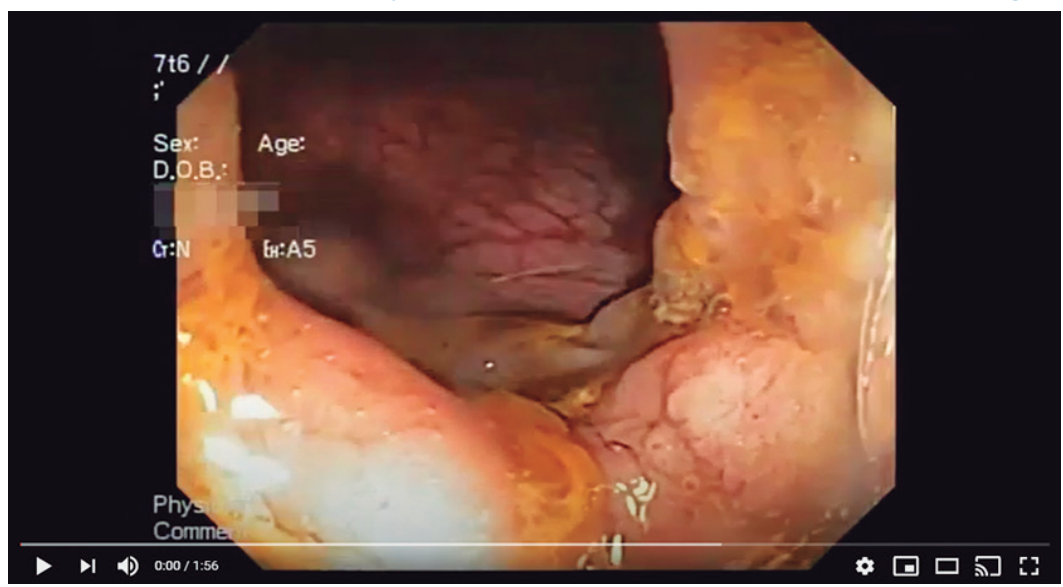


Figura 2. Anatomía patológica de los cortes en hematoxilinaeosina
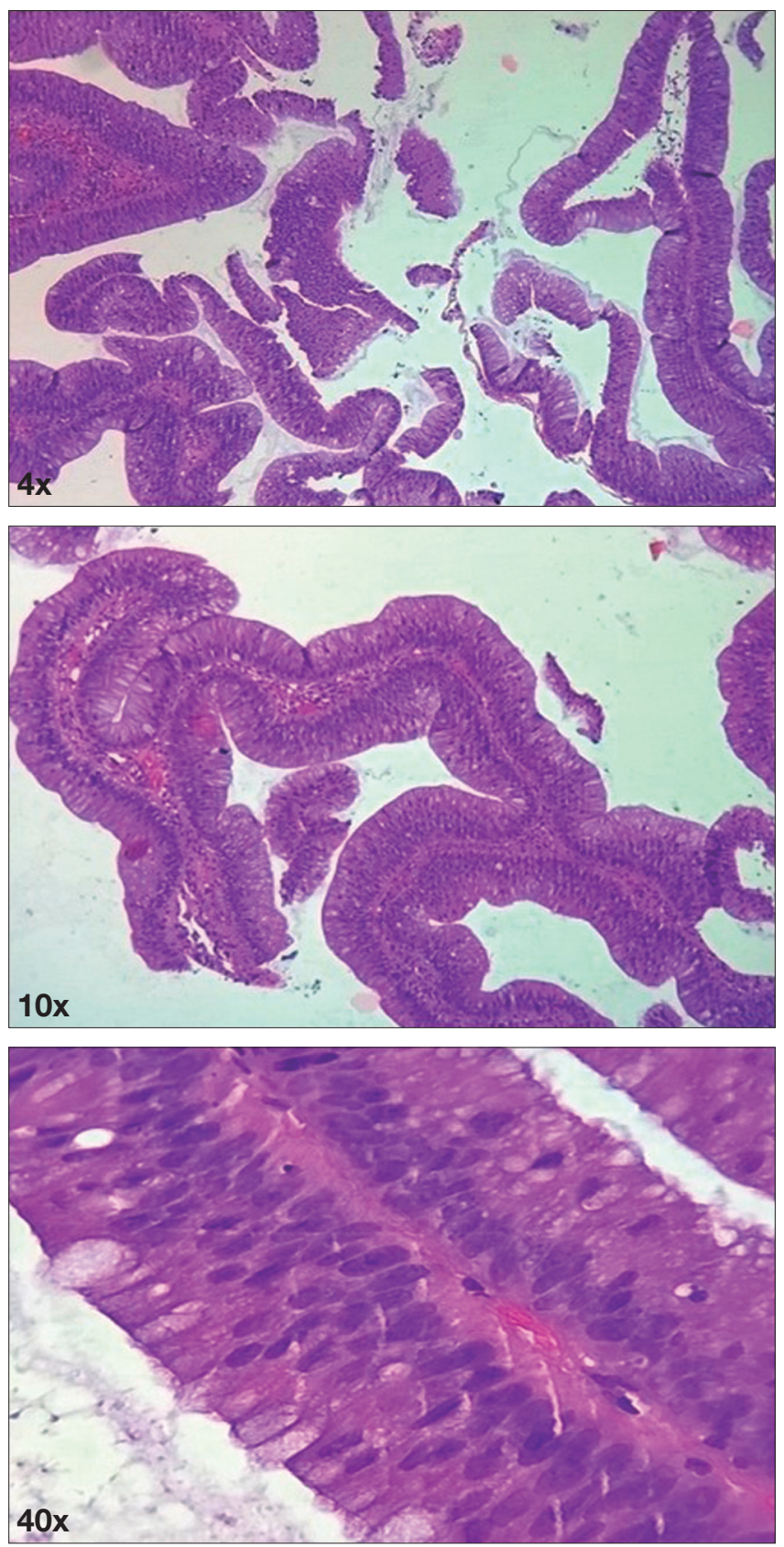

Procesos digitiformes constituidos por el tejido conectivo recubierto de las células epiteliales. Estas presentan displasia de bajo grado con pseudoestratificación del epitelio glandular, con núcleos elongados, hipercromáticos, localizados de forma predominante en la porción basal y escasa actividad mitótica.

Para evaluar el compromiso ganglionar y de los órganos adyacentes, se solicitó una tomografía del abdomen y pelvis, sin encontrar hallazgos patológicos.

Se diagnosticó el SMcW por la presencia de un adenoma velloso gigante del recto con diarrea crónica, una insuficiencia renal y un desbalance hidroelectrolítico. Inicialmente se corrigió el desbalance hidroelectrolítico, la injuria renal y se realizó un tratamiento médico de la diarrea secretora.

Como tratamiento definitivo, se optó por la resección quirúrgica como la opción más adecuada por el tamaño tumoral, la forma circunferencial y las dificultades técnicas endoscópicas que conlleva la exéresis de este tipo de lesiones. El paciente no aceptaba un estoma definitivo, por lo que se le realizó una cirugía con la técnica en dos tiempos conocida como pull through con anastomosis coloanal. El estudio de la pieza quirúrgica informó macroscópicamente una lesión extensa de 130 x 90 mm elevada con aspecto velloso. En el estudio histológico se estudiaron 102 fragmentos de la lesión descripta y se arribó al diagnóstico de adenoma velloso con displasia de bajo y alto grado sin imágenes de invasión en el material examinado (Figura 3).

En el posoperatorio se detectó un absceso pélvico que requirió un drenaje percutáneo, con una evolución ulterior favorable.

Al momento del alta, el paciente presentaba las deposiciones formadas, la función renal y el ionograma sin alteraciones y buena continencia para la materia fecal y los gases.

\section{Figura 3. Anatomía patológica de la pieza quirúrgica}
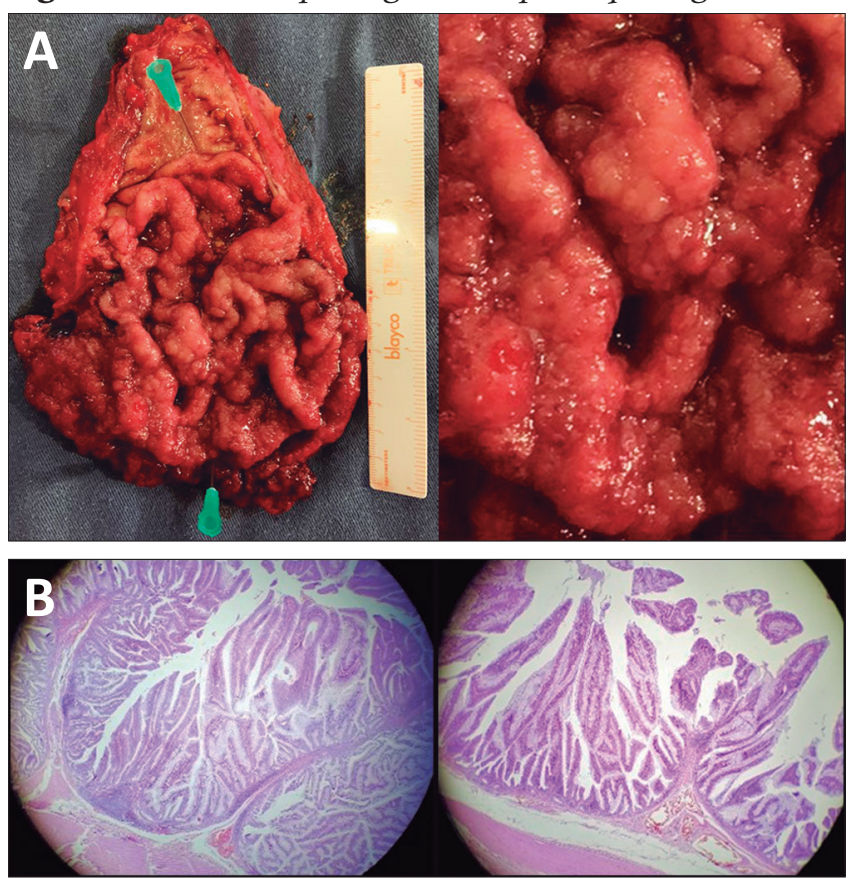

A: Macroscópicamente se observa una lesión de 130 × 90 mm sobreelevada con aspecto velloso que engrosa los pliegues.

B: Microscópicamente se confirma el diagnóstico del adenoma velloso con una displasia de bajo y alto grado sin sectores de invasión. 


\section{Discusión}

Se presentó el caso clínico de un paciente con diarrea crónica, con repercusión hidroelectrolítica e insuficiencia renal asociadas a LST circunferencial de recto con resultado histológico de adenoma velloso con displasia de bajo grado. Se le realizó el diagnóstico del SMcW, entidad que puede generar severas complicaciones con una elevada mortalidad. ${ }^{7}$ Este síndrome, descripto por primera vez en 1954 por Leland McKittrick y Frank Wheelock, está presente en el $2 \%$ de los adenomas vellosos siendo más frecuente en aquellos que presentan un diámetro mayor a $40 \mathrm{~mm}$. Estos adenomas secretan grandes cantidades de sodio y potasio; pueden causar injuria renal aguda que en casos extremos requieran hemodiálisis. Además, como consecuencia de la depleción hidroelectrolítica, clínicamente pueden presentar vómitos, confusión, síncope, astenia y arritmias. 5

Al inicio del cuadro, la pérdida hidroelectrolítica es compensada por la ingesta y los mecanismos de regulación renal. Cuando el tumor crece, las pérdidas superan la compensación, lo que resulta en pérdidas entre 1,5 a 3,5 litros diarios que contienen en promedio $40-160 \mathrm{mmol} / \mathrm{l}$ de sodio, $15-105 \mathrm{mmol} / \mathrm{l}$ de potasio y $80-165 \mathrm{mmol} / \mathrm{l}$ de cloro. ${ }^{8}$

La hipersecreción de agua y sodio de las células adenomatosas de los tumores vellosos se origina por una producción aumentada de prostaglandina E2 (PGE2) que actúa como secretagogo y produce diarrea secretora. El aumento de esta prostaglandina, así como su disminución luego de la resección del adenoma, se demostró en las muestras de materia fecal. $5,6,8$

Los pacientes que presentan adenomas vellosos proximales mantienen un balance de sodio normal, mientras que cuando los adenomas están presentes cerca del ano no permiten una reabsorción efectiva de este ion. ${ }^{5}$

En los pacientes que presentan la tríada diagnóstica de diarrea crónica, falla prerrenal y alteración hidroelectrolítica se recomienda realizar una VCC. ${ }^{8}$

Hay que tener en cuenta que el diagnóstico de esta enfermedad es complejo, ya que presenta un curso insidioso, síntomas poco específicos y una baja incidencia, lo que conlleva un retraso diagnóstico y terapéutico que empeora el pronóstico.

Una vez establecido el diagnóstico del SMcW, su posterior tratamiento debe tener un enfoque multidisciplinario. La elección del tratamiento definitivo debe ser individualizada, considerando la seguridad, la aplicabilidad y la eficacia del método.

Para el control de la diarrea, se ha demostrado que el uso de indometacina, un inhibidor de la secreción de PGE2, disminuye el componente secretor del adenoma y las pérdidas iónicas. Es eficaz hasta en el 50\% de los pacientes. 5,8

El tratamiento definitivo de este síndrome es la resección del tumor, ya que resuelve la diarrea, la alteración hidroelectrolítica y evita la progresión a adenocarcinoma. Actualmente, hay dos alternativas: la resección por vía endoscópica o la quirúrgica.

El manejo se puede realizar tanto por la resección endoscópica mucosa (REM) o por la disección endoscópica submucosa (DES). Ambas técnicas se han asociado a un alto porcentaje de éxito: entre 85 y $95 \%{ }^{9}$

Un factor a favor para realizar la resección por vía endoscópica de los LST rectales es el bajo riesgo de invasión submucosa de este tipo de lesiones, lo que permite que sea una alternativa válida en la mayoría de los casos según varias series. ${ }^{10}$

En la actualidad, hay interés respecto al uso de la ultrasonografía endoscópica (USE) a nivel rectal para valorar la profundidad de la invasión de los LST, lo que posibilitaría el tratamiento endoscópico por la REM o la DES, evitando los tratamientos quirúrgicos innecesarios. ${ }^{11-13}$

Con respecto a la REM, la resección en bloque es la opción ideal. Cuando no es posible técnicamente, se puede realizar la resección en fragmentos con mayor riesgo de adenoma residual de hasta el $15 \%$ en algunas series. ${ }^{14}$ Por este motivo, se sugiere un seguimiento endoscópico a intervalos (3, 6, 12 y 24 meses) luego de la resección en fragmentos. ${ }^{2}$

La DES logra una resección más profunda, siendo eficaz en las lesiones con elementos sugestivos de invasión submucosa. Es una técnica compleja, lo que limita su uso en este tipo de lesiones. ${ }^{9,14}$

La evidencia disponible señala el alto rendimiento que tiene el manejo endoscópico en lesiones mayores de $20 \mathrm{~mm}$, pero no existen hoy por hoy ensayos clínicos en lesiones de gran tamaño, como el caso del paciente en discusión que presentó una lesión circunferencial de $180 \mathrm{~mm}$. Se dispone únicamente de casos reportados de adenomas rectales gigantes como el de Roriz-Silva et al. y el de Agnes et al., que presentaron un tamaño tumoral de $100 \mathrm{~mm}$ y $150 \mathrm{~mm}$ respectivamente. En estos casos, los tumores se extirparon quirúrgicamente. ${ }^{15,16}$ No existe un punto de corte claro en cuanto al tamaño del tumor para definir cuáles son pasibles de tratamiento endoscópico.

En vistas a la resección endoscópica, las características del tumor en el caso presentado le conferían (por su topografía próxima al margen anal, tamaño y forma circunferencial) gran complejidad técnica. Asimismo, su tamaño y su forma generaban un alto riesgo de perforación, estenosis y sangrado. Por esto se decidió realizar un tratamiento quirúrgico con la finalidad de 
lograr la resección completa con los criterios de resección oncológicos, debido a la alta asociación $(80 \%)$ con focos microscópicos de adenocarcinoma en adenomas vellosos extensos (carpet villous adenoma) que, en general, son estadios iníciales T1 o in situ. ${ }^{7}{ }^{17}$ La técnica a utilizar (que puede abarcar desde técnicas transanales, resecciones bajas o ultrabajas de recto con técnicas de conservación esfinteriana hasta la amputación abdominoperineal) dependerá de la extensión distal de la lesión, la relación con el canal anal, el estado general del paciente, el estado funcional del esfínter y los deseos y expectativas del paciente dado la posibilidad de necesitar una colostomía definitiva.

Los abordajes transanales en los últimos años han avanzado, destacándose la TEM, la TAMIS y, recientemente, la TaTME. Sin embargo, su indicación en el manejo del SMcW, aunque mostrando resultados favorables, ha quedado limitada a los centros de alto volumen de patología colorrectal. ${ }^{7} 18$

En la actualidad, el gold standard es la vía anterior tanto por abordaje abierto como laparoscópico.

En el presente caso, la lesión alcanzaba la línea pectínea en el canal anal quirúrgico y ascendía hasta la unión rectosigmoidea. El paciente presentaba una buena función esfinteriana y no aceptaba un estoma definitivo, por lo que se optó por la técnica pull through con anastomosis coloanal. La misma consiste en una proctectomía con la preservación esfinteriana en 2 etapas. La primera cirugía consiste en una escisión mesorrectal total por el abordaje combinado abdominal y perineal. En el abordaje abdominal laparoscópico, se realiza la disección mesorrectal hasta el elevador del ano. El abordaje perineal completa la disección distal, seccionando la pared anorrectal al nivel de la línea pectínea, con la extracción del espécimen y la exteriorización del cabo sigmoideo transanal (pull through). Se deja un cabo de $10 \mathrm{~cm}$ de sigmoides al cual se le realizan 2-4 puntos de anclaje a la piel.

A los 7 días se completa la resección del segmento sigmoideo exteriorizado y se realiza la anastomosis coloanal de forma manual. Las ventajas de esta técnica radican en la conservación esfinteriana, la ausencia de la colostomía definitiva y la realización de una anastomosis coloanal sin necesidad de una ostomía temporaria de protección para la anastomosis.

En el posoperatorio se detectó un absceso pélvico (una complicación descrita para esta cirugía en un porcentaje cercano al 10\%) que requirió un drenaje percutáneo, con una evolución ulterior favorable. Al momento del alta, el paciente presentaba deposiciones formadas, la función renal y el ionograma sin alteraciones y una buena continencia para las materias y los gases.

\section{Conclusión}

El síndrome de Mckittrick Wheelock es una causa poco frecuente de diarrea crónica con alteración hidroelectrolítica, causado por un tumor rectal hipersecretor con un diámetro mayor a los $40 \mathrm{~mm}$. El tratamiento inicial consiste en la reposición hidroelectrolítica, el manejo de las complicaciones y la resección del tumor como manejo definitivo, ya sea por vía endoscópica o quirúrgica. La decisión del manejo debe individualizarse según las características de la lesión, junto con la disponibilidad y el entrenamiento del personal médico. La exéresis del tumor resuelve la diarrea y sus complicaciones, además de prevenir la progresión a un adenocarcinoma.

\section{Sostén financiero. Para la realización del presente trabajo no se recibieron aportes financieros.}

Conflictos de intereses. Ninguno.

\section{Aviso de derechos de autor}

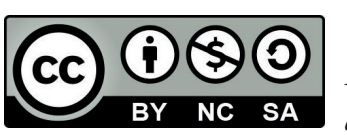

(C) 2021 Acta Gastroenterológica Latinoamericana. Este es un artículo de acceso abierto publicado bajo los términos de la Licencia Creative Commons Attribution (CC BY-NC-SA 4.0), la cual permite el uso, la distribución $y$ la reproducción de forma no comercial, siempre que se cite al autor y la fuente original.

Cite este artículo como: Leites $M$, Canavesi A, Valencia $D$ y col. Resección de adenoma velloso gigante causante del sindrome de Mckittrick-Wheelock. Acta Gastroenterol Latinoam. 2021;51(1):93-99. https://doi.org/10.52787/ rwop3269

\section{Referencias}

1. Cáceres Fluidio G, González Flaqué N. Pólipos Colorrectales. Clin Quir Fac Med UdelaR. 2018:1-7.

2. Hurlstone DP, Sanders DS, Cross SS, et al. Colonoscopic resection of lateral spreading tumours: a prospective analysis of endoscopic mucosal resection. Gut. 2004;53(9):1334-9.

3. Zhao X, Zhan Q, Xiang L, Wang Y, Wang X, Li A, Liu S. Clinicopathological characteristics of laterally spreading colorectal tumor 2014;9(4):e94552.

4. Horiuchi Y, Chino A, Matsuo Y, et al. Diagnosis of laterally spreading tumors (LST) in the rectum and selection of treatment: characteristics of each of the subclassifications of LST in the rectum. Dig Endosc. 2013;25(6):608-14.

5. Mila R, Grille S, Laurini M, Lapiedra D, Bagattini JC. Síndrome de McKittrick-Wheelock: Una causa infrecuente de shock hipovolémico. Rev Med Chil. 2008;136(7):900-4. 
6. Van der Pool AEM, de Graaf EJR, Vermaas M, Barendse RM, Doornebosch PG. McKittrick Wheelock Syndrome Treated by Transanal Minimally Invasive Surgery: A Single-Center Experience and Review of the Literature. J Laparoendosc Adv Surg Tech A. 2018;28(2):204-8.

7. Kral A, Vega J. Síndrome de Mckittrick-Wheelock. Una causa infrecuente de hipokalemia e injuria renal aguda. Caso clínico. Rev Med Chil. 2017;145(7):950-3.

8. Bruno A, Chimienti D, Montanaro A, et al. The mckittrick-wheelock syndrome: a rare cause of severe hydroelectrolyte disorders and acute renal failure. Case Rep Nephrol. 2011;2011:765689.

9. Stroppa I, Milito G, Lionetti R, et al. Rectal laterally spreading tumors successfully treated in two steps by endoscopic submucosal dissection and endoscopic mucosal resection. BMC Gastroenterol 2010;10:135.

10. Buchner AM, Guarner-Argente C, Ginsberg GG. Outcomes of EMR of defiant colorectal lesions directed to an endoscopy referral center. Gastrointest Endosc 2012;76(2):255-263.

11. Hasak S, Kushnir V. Rectal Endoscopic Ultrasound in Clinical Practice. Curr Gastroenterol Rep 2019;21(4):18.

12. Schizas AM, Williams AB, Meenan J. Endosonographic staging of lower intestinal malignancy. Best Pract Res Clin Gastroenterol. 2009;23(5):663-70.
13. Worrell S, Horvath K, Blakemore T, Flum D. Endorectal ultrasound detection of focal carcinoma within rectal adenomas. Am J Surg. 2004;187(5):625-9.

14. Ferlitsch M, Moss A, Hassan C, et al. Colorectal polypectomy and endoscopic mucosal resection (EMR): European Society of Gastrointestinal Endoscopy (ESGE) Clinical Guideline. Endoscopy. 2017;49(3):270-97.

15. Roriz-Silva R, Andrade AA, Ivankovics IG. Giant rectal villous adenoma: Surgical approach with rectal eversion and perianal coloanal anastomosis. Int J Surg Case Rep. 2014;5(2):97-9.

16. Agnes A, Novelli D, Doglietto GB, Papa V. A case report of a giant rectal adenoma causing secretory diarrhea and acute renal failure: McKittrick-Wheelock syndrome. BMC Surg. 2016;16:39.

17. Rutter MD, Nickerson C, Rees CJ, et al. Risk factors for adverse events related to polypectomy in the English Bowel Cancer Screening Programme. Endoscopy. 2014;46(2):90-7.

18. Orchard MR, Hooper J, Wright JA, McCarthy K. A systematic review of McKittrick-Wheelock syndrome. Ann R Coll Surg Engl. 2018;100(8):1-7. 